\title{
A History of the Epilepsy Programme at University Hospital (LHSC) \& Western University, London, Ontario Canada 1975- 2012
}

\author{
Warren T. Blume, John P. Girvin
}

\section{Prologue: Epidemiology of Intractable Epilepsy \& Temporal Lobe Epilepsy}

With a Canadian 1975 population of 20 million (Statistics Canada) and an epilepsy prevalence of six per thousand ${ }^{1}$, about 120,000 Canadians had chronic epilepsy in 1975. Epilepsy resistant to anti-epileptic drugs (AEDs) afflicts one third of all patients with epilepsy, thus about 40,000 Canadians suffered from further seizures despite treatment. Engel ${ }^{2}$, using data from that era, estimated that $10 \%$ of all epilepsy patients "might benefit from surgical intervention", thus 12,000 Canadians!

As temporal lobe epilepsy (TLE) prevalence was estimated to be 1.7 per thousand ${ }^{1}, 34,000$ had TLE in 1975. The temporal lobe, the brain's most epileptogenic area, tends to resist AEDs; thus $\sim 11,000$ Canadians in 1975 felt the burden of recurrent temporal lobe seizures. Theoretically, all of these 11,000 patients could have been evaluated for epilepsy surgery.

Despite these compelling data, no epilepsy surgery centre existed in Ontario in the 1970s.

\section{A Fortuitous Beginning of our Epilepsy Programme}

In early 1974 Italian parents brought their eight year old son with intractable regional right hemisphere epilepsy to University Hospital London to learn whether the world-famous Dr. Charles Drake could improve his condition by resective surgery. As Dr. Drake operated very principally on vascular conditions, he referred the patient to Dr. John P. Girvin (JPG) who had trained at the Montreal Neurological Institute and received a $\mathrm{PhD}$ in neurophysiology from McGill University. Having trained in epileptology and electroencephalography (EEG) in Montreal and the Mayo Clinic, Dr. Warren Blume (WTB) became involved in evaluating this boy's seizure disorder for its medical and surgical management. Our educational backgrounds having complemented each other for epilepsy, we continued our teamwork approach for other patients. That the first three operated patients had extra-temporal, extra-limbic seizure disorders paradoxically sharpened our distinction of temporaloriginating epilepsies from those propagating to the temporal lobe from other sites. Such temporal-propagated seizures often originated in the occipital lobe. Dr. Girvin's considerable scientific and surgical experience in the visual cortex enabled us to evaluate and excise epileptogenic tissue in an area avoided by most epilepsy surgery centres ${ }^{3}$.

\begin{abstract}
Abilities that Complement
Lacking neuroimaging, such as magnetic resonance imaging (MRI) at that time, surgical decisions - whether to operate, what area of cortex, and extent - depended upon ictal semiology, neurological examination, and EEG. On admission to University Hospital, we (WTB \& JPG) each obtained seizure and neurological history and neurological examination independently, then compared the results. This spawned an exciting, friendly rivalry to determine which of us could obtain the more cogent, intricate and thorough ictal data from the patient and family, thus useful and dependable in reaching decisions. In this process, each taught the other with help from residents and fellows. To this day, ictal semiology remains the principal factor in formulating possible surgical management.

To supplement our knowledge of the basic science aspect of epileptology, JPG \& WTB held a two- person journal club that met at $0700 \mathrm{hrs}$, three days per week, in the summer of 1977, when the majority of chapters in Basic Mechanisms of the Epilepsies (1969) were individually covered. This type of concentrated interaction efficiently and beneficially introduced us as new colleagues to each other.
\end{abstract}

\section{Epilepsy Programme at University of Western Ontario (UWO)}

The Epilepsy Programme at UWO (now Western University) began formally in 1977 with an Academic Development Fund award to WTB and JPG. This was supplemented by a generous donation from the Richard and Jean Ivey Fund. With this impetus, London became the second major centre in Canada devoted to intractable epilepsy after the Montreal Neurological Institute. Subsequent support arose from the JP Bickell and PSI foundations, Mr. Ed Aitken, and the London Life Insurance among others.

Our programme would not have been realized without the guidance and continued support of Dr. HJM Barnett, Chairman,

\footnotetext{
From the Department of Neurology, Western University, University Hospital, London, Ontario, Canada.

Received January 26, 2012. Final Revisions Submitted August 7, 2012. Correspondence to: Warren T. Blume, Neurology, Western University, University Hospital, 339 Windermere Rd, B10-118, London, Ontario, N6A5A5, Canada. Email: Warren.blume@lhsc.on.ca.
} 
Department of Clinical Neurological Sciences, UWO and Mr. Pat Blewett, President and Chief Executive Officer, University Hospital.

\section{Epilepsy/EEG Fellows}

Drs. G. Bryan Young and Richard S. McLachlan, both having received their FRCPC in neurology, elected to become our first epilepsy-EEG fellows in 1977 and 1978 respectively. Subsequently invited to join our staff, each has provided significant contributions to patient care and academic achievement in our programme over the years. Thirty-five epilepsy-EEG fellows have received training in our programme of whom over ten have become chairpersons of their own programmes.

\section{Epilepsy Surgeons}

As a neurosurgeon and neurophysiologist, Dr. John Girvin provided an outstanding model as an epilepsy surgeon that attracted several young neurosurgeons to our programme over the years. In chronological order these have been: Drs. Howard Reichman, Andrew Parrent, Ramesh Sahjpaul, David Steven, Sandrine deRibeaupierre, and Keith MacDougall.

\section{Epilepsy In-Patient Units: 1985, 1993}

As the benefits of inpatient assessment of patients became recognized, an increasing number of beds on the neurology floor were occupied by epilepsy patients, each with a bulky, sharpcornered EEG machine. With the inevitably incomplete surveillance on a ward serving multiple neurological disciplines, it is likely that some seizures remained unobserved and may not have received adequate care. Moreover, most neurological nurses had not obtained the subspecialty training and experience needed to optimally manage patients with intractable epilepsy.

Therefore, under the guidance and support of Mr. Pat Blewett and Ms. Diane Stewart, Executive Vice President of University Hospital, a four-bed communal Epilepsy Unit with 24/7 nursing surveillance and care, along with continuous EEG monitoring, opened in December 1985. Dr. Pierre Gloor of the MNI, was our distinguished invited guest at the official opening ceremonies in January 1986.

Eventually, the waiting list for the 4-bed unit became unacceptably long leading to the current 8-bed unit opening in 1993 with Dr. Juhn Wada as guest of honour. Our continuous monitoring was further advanced by video seizure monitoring of all eight patients.

\section{Nursing in the Epilepsy Unit}

Our inpatient unit could not have functioned without caring and knowledgeable nurses; thus a subspecialty of Epilepsy Nursing developed within Neurological Nursing. These nurses tend to the needs of patients as they observe and document seizure activity. Safety is a priority for patients, nurses and equipment during seizures. They also detect incipient status epilepticus (SE) to prevent its development. Among patients with TLE, emergence of increasing motor components may herald SE. Margaret Hamilton, RN, created a manual as a guide for inpatient nursing care of epilepsy patients ${ }^{4}$.

\section{Evaluation of Patients for Epilepsy Surgery}

An offer of surgery has been typically given to the patient and to her/his closest relative or friend in an interview given by both JPG and WTB together, to emphasise our team concept of care. A joint interview assured that a coherent, thus clear description was presented of: 1) our rationale, 2) favourable and unfavourable aspects of the epilepsy condition specific for that patient that relate to the proposed procedure, 3) goals and possible outcomes with likelihood of each and 4) minor and major surgical-related complications. For the past several years each surgical candidate is discussed by our entire epileptology team of neurologists and neurosurgeons.

In 1992, JPG described the pathophysiology and time course of transient and permanent deficits resulting from resective surgery ${ }^{5}$. These specifics of operative and medical/surgical postoperative care kept the patient, relatives and all team members informed.

Over the first ten years of the Epilepsy Programme our surgically operated patients quintupled - from 11to 55 per year while about three times that number of intractable epilepsy patients were evaluated for surgery. Our waiting list grew to 85 patients by 1988 of whom $38 \%$ came from southwestern Ontario, 32\% elsewhere in Ontario, 17\% from other Canadian provinces and $13 \%$ from other countries. These intractable epilepsies arose from a temporal lobe in more patients than those from other lobes.

\section{Electroencephalography (EEG)}

Electroencephalography has remained a cornerstone of epilepsy surgery evaluation, even with the advent of the equally valuable MRI. To maximize the precision and reliability of EEG requires exemplary EEG technology, thus superb technologists. During our evaluations of the first four cases (above), Mr. D. Kent McNeill and Ms. Peggy Stewart each demonstrated that our burgeoning centre had EEG capability "in spades". Each trained in London, UK: Kent at Saint Bartholomew's Hospital and Peggy at the Maudsley Hospital. It is doubtful that we would have proceeded to develop an epilepsy programme without this component. Our resulting exemplary EEG standards soon attracted other exceptional technologists, notably Mr. Dan C. Jones and Mrs. Masako Kaibara. Today the precise technical quality of EEG has been maintained by current EEG techologists: Janet Demelo, Giannina Holloway, Cathy Johnson, Neda Lubus, Jennifer Rosser, Elyse Sandison, Ashleigh SmithAdam and Linda Vaillancourt. Giannina Holloway co-authored with Dr. GB Young and Masako Kaibara our most recent EEG Atlas $^{6}$.

\section{Subdural EEG}

In 1979, Dr. John Girvin and Mr. Dan Jones, EEG technologist, designed subdural electrodes. Inserted as imbedded in silastic strips through burr holes to avoid a craniotomy during the patient's evaluation, these electrodes record directly from the cortical surface. Moreover, they may extend to mesial and inferior cortical surfaces, areas remote from scalp electrodes. The initial electrodes failed to have the flexibility, the ideal metal, and a satisfactory series of recording contacts on each of the electrode strips. These deficiencies were, 
over the course of the following years, continually altered until satisfactory modifications had been achieved. The alterations in the electrodes allowed extension to mesial and inferior cortical surfaces, areas remote from scalp electrodes.

Commercially manufactured subdural electrodes were not available in the early 1980's, resulting in some other epilepsy centres purchasing our in-house-manufactured electrodes. The design and manufacture of these electrodes, at UH without any complication, continued by Dan Jones and Frank Bihari for over 20 years.

However, in 2004 London Health Sciences Centre chose to make use of commercially-produced electrodes, though at a significantly greater cost.

Our centre was the first to routinely employ subdural electrodes (SDE) and they continue today as a major part of evaluation of about $50 \%$ of our patients ultimately operated upon.

For patients with temporal lobe epilepsy, SDE provides more precise and sensitive ictal and interictal recording from the mesial temporal regions. We studied 27 consecutive patients whose temporal lobe epilepsy clinically implicated both temporal lobes from ictal semiology, scalp EEG and imaging features. We found that the side of SDE-recorded seizures correlated with that containing most scalp spikes and most scalprecorded seizures in most but not all patients, confirming the value of both EEG and $\mathrm{SDE}^{7}$. Dr. David Diosy was among our members who contributed to a study on the SDE role in determining whether and in what area epilepsy surgery should be performed $^{8}$.

Frank Bihari also has been integral in the development of several types of electrodes for intra-cerebral recording, among which have been: double-sided subdural paddle electrodes, intraventricular electrodes and a split cannula for implantation. He also helped develop a 3D computer reconstruction of brain mapping for resective surgery through innovative software.

\section{Electrocorticography (ECoG)}

Electrocorticography, routinely recorded in the years before SDE and MRI, helped to confirm localization of epileptogenesis disclosed by ictal semiology, interictal spikes and delta activity ${ }^{9}$, and scalp-recorded seizures ${ }^{10}$. Although we EEGers can recognise spikes, a more difficult task is estimating their relative quantity among various regions. A computer program developed in our EEG laboratory provided and displayed such spike quantities among recorded cortical regions ${ }^{11}$. Post-resection recordings were ultimately eliminated for temporal lobe patients as post-resection spike quantity failed to correlate with outcome $^{12}$. Subsequently, SDE and MRI lessened the need for $\mathrm{ECoG}$ in many cases, particularly those of temporal lobe origin.

\section{Obtaining and Analysing EEG Data}

To overcome the time limitations of standard EEG recordings required by EEG telemetry, thus 24/7 capability, Mr. John Ives, a Queens University graduate in engineering, then working at the Montreal Neurological Institute, designed, made and faithfully maintained our first EEG telemetry system in 1983. An EEG montage that accompanied his system has been named "The Ives Montage" in perpetuity. The vast quantity of data pouring out from this and subsequent telemetry systems required selection by EEGers, as well as classification and storage in a database with search capability. Mr. John Lemieux, our computer system analyst from 1980 to his passing in 2002, wrote programs to this effect. Additionally, his field plot computer program disclosed that "generalised" spike-waves often originate in one cerebral hemisphere - providing insight into the relationship between focal and generalised epilepsy ${ }^{13}$.

\section{Magnetic Resonance Imaging (MRI)}

A second major advance in evaluating patients for temporal lobe surgery was the advent of MRI. Imaging afforded by MRI, disclose focal structural abnormalities underlying intractable epilepsy that remained undetected by earlier neuroimaging methods ${ }^{14}$. Subsequently, Lee et al demonstrated both high sensitivity and specificity for MRI in detecting pathologicallyverified hippocampal/amygdala and other temporal lobe epileptogenic lesions ${ }^{15}$.

\section{Functional Magnetic Imaging (fMRI)}

Dr. Seyed Mirsattari's group has employed fMRI to map the effects of temporal lobectomy on neuropsychological functioning ${ }^{16}$. Seyed Mirsattari, John Ives and others also fostered development of MRI-compatible EEG electrodes which obviated the need to remove scalp electrodes for MRI in Epilepsy Unit patients undergoing 24/7 monitoring ${ }^{17}$.

\section{Neuropsychology \& the Wada Test of Language and Memory}

Our highly capable neuropsychology team, now led by Drs. Brent and Susan Hayman-Abello continue to generate reliable and pertinent clinical information, especially involving language and memory obtained by standard neuropsychological testing. Previous neuropsychologists included Dr. Jeannette McGlone and Dr. Michael Harnadek.

In the test created by Dr. Juhn Wada in publications from 1949 to 1960 , language function and memory of each cerebral hemisphere is tested while the opposite hemisphere is transiently anesthesised by carotid injection of either amytal or etomidate. The Hayman-Abellos successfully piloted the transition from sodium amytal to etomidate as the drug administered separately to each hemisphere. This transition over the past few years was prompted by unavailability of amytal in Canada.

\section{Epilepsy in Neurocritical Care}

Medically refractory seizures may complicate the course of many neurological and non-neurological disorders. Thanks to Dr. G. Bryan Young, our world-renowned neurocritical care specialist for over three decades, our Epilepsy Programme extends to this area as well ${ }^{18,19}$.

\section{Brain Electrical Stimulation for Epilepsy}

Drs. McLachlan and Parrent and associates have sought improved seizure control via hippocampal and via vagus nerve electrical stimulation and have comprehensively published results thereof ${ }^{20-22}$. 


\section{Follow-Up Studies \& Quality of Life}

Many studies have demonstrated significantly improved seizure control as a result of temporal lobectomy, but these studies had not distinguished the relative contributions of the surgery itself and further medical management to this amelioration.This requires a randomized, controlled trial (RCT) in which candidates for temporal lobectomy randomly enter a medical or surgical arm for a predetermined period - a daunting challenge for any epilepsy surgery investigator, the patients, and the centre involved.

Dr. Samuel Wiebe, a former staff member in our Programme, astonished the epileptology world and gained universal high praise for accomplishing this epidemiology/neurology feat as described in his landmark New England Journal of Medicine article demonstrating 58\% seizure freedom in the surgical group and $8 \%$ in the medical $\mathrm{arm}^{23}$. To date this study remains the only completed RCT in the world investigating the results of surgery in the management of temporal lobe epilepsy.

Several of our projects have investigated quality of life and mental health of our patients, including effects of temporal lobectomy. Psychiatric disorders were diagnosed in almost half of candidates for epilepsy surgery by Dr. R. Manchanda, formerly our Epilepsy Programme psychiatrist, using DSM-III$\mathrm{R}$ criteria. Among these, anxiety and personality disorders were the most common.Using the Epilepsy Surgery Inventory 55 (ESI-55), McLachlan et al, found significantly improved Health Related Quality of Life (HQOLIE) among patients rendered seizure-free or $>90 \%$ less seizures ${ }^{24,25}$. However, HQOLIE deteriorated in those with less favourable outcomes. Dr. Kathy Speechley' group has studied the impact of childhood epilepsy upon families and found depression in both mothers and fathers $^{26-28}$.

Stereotactic amygdalohippocampotomy, by placing a series of destructive lesions in the amygdala and 13-54 $\mathrm{mm}$ of the anterior hippocampus, gave favourable outcomes in $9(60 \%)$ of 15 patients so treated at our centre ${ }^{29}$, thus somewhat less effective than a standard temporal lobectomy. Smaller ablations were ineffective.

Surgical effectiveness among patients whose pre-operative data were sufficiently complex to require SDE to localize seizure onset was favourable as disclosed by a follow-up study by Dr. Keith MacDougall and colleagues ${ }^{30}$.

\section{Basic Science}

Recognising that studies of related cellular neurophysiology could clarify many complexities of human epilepsy, our Epilepsy Programme fortunately includes two world class neurophysiologists in Drs. Stan Leung and Michael Poulter. Among other interests, Dr. Leung has studied hippocampal (HPC) theta thythms and Dr. Poulter has extensively investigated HPC inhibition.

\section{Antiepileptic Drug (AED) Trials}

Although older AEDs never lose their roles in epilepsy patient care, the advent of a new drug always brings with it renewed hope for better seizure control. Suzan Brown, our Clinical Research Coordinator, is thus able to enroll some patients in formal pre-market evaluation of newly-developed
AEDs. In an attempt to attract new drug trials to London as readily as they are to European and American centres, JoAnne DePace, a previous Coordinator, and WTB developed a comprehensive booklet, Bridging the gap; epilepsy pharmaceutical research (1999), illustrating our programme's many abilities to clinically and scientifically evaluate potential AEDs. However, Canada (thus London) still trails Europe and the US in AED trial sequence.

\section{Epilepsy Programme-Hosted Conferences}

The LHSC Epilepsy Programme has organized two Canadian League Against Epilepsy conferences in London, Ontario. In 1997, JPG and WTB convened the first Canadian Temporal Lobe Epilepsy Surgery workshop. This brought together epileptologists from across Canada to discuss the role of resective surgery in TLE management includingits pitfalls and limitations ${ }^{31}$. In 2004 our group hosted a conference on the multiple aspects ofIntractable Epilepsy, with Canadian and international participants ${ }^{32}$. A principal section of this conference centred on TLE.

\section{Epilepsy Support Centre}

Recognising that persons with epilepsy are often isolated, unemployed and stigmatized, this centre offers many programs for people of all ages. Founded in the 1970s by a group including Mr. and Mrs. William (Bill) Holmes and now ably directed by Mary Secco, this centre has thrived over the years despite much less financial support than received by similar lay organisations of other neurological disorders.

\section{THE Future}

Now under the inspired co-leadership of Dr. Jorge Burneo and Dr. David Steven, our group comprises an effective blend ofthe experienced and of youth, each exuberant and wise. Collective interests range from patient care (a uniform priority) to academia, interests that complement, but do not clash. We communicate easily, flexibly and fruitfully.

Dr. Burneo will continue his role in the Provincial Epilepsy Network Group in its efforts to improve access to epilepsy care in Ontario. He and Dr. David Steven also have created a programme supporting provision of medical and surgical care of epilepsy in Peru. Dr. Steven and Dr. Burneo will continue to apply their training in biostatistics and epidemiology to surgical outcomes as related to the various epilepsy etiologies and evaluation techniques ${ }^{33}$.

As the co-presence of adult and paediatric epilepsy units mutually benefits each, we are fortunate to anticipate the continued emergence of our partner programme at the Children's Hospital of Western Ontario under the enthusiastic efforts of neurologists Drs. Simon Levin, Narayan Prasad and Craig Campbell; neurosurgeon, Dr. Sandrine de Ribaupierre; and nurse practitioner Ms. DeVries-Rizzo. 


\section{REFERENCES}

1. Hauser WA, Kurland LT. The epidemiology of epilepsy in Rochester, Minnesota, 1935 through 1967. Epilepsia. 1975;16: $1-66$.

2. Engel JJ, Shewmon DA. Who should be considered a surgical candidate? In: Engel JJ, ed. Surgical Treatment of the Epilepsies. New York: Raven Press; 1993:23-34.

3. Blume WT, Whiting SE, Girvin JP. Epilepsy surgery in the posterior cortex. Ann Neurol. 1991;29:638-45.

4. Hamilton M. Epilepsy Monitoring Unit Teaching Manual. In: Center LHS, ed. London; 2011.

5. Girvin JP. Complications of Epilepsy Surgery. In: Luders HO, ed. Epilepsy Surgery. New York: Raven; 1992:653-60.

6. Blume WT, Holloway GM, Kaibara M, Young GB, eds. Blume's Atlas of Pediatric and Adult Electroencephalography. Philadelphia: Lippincott Williamas \& Wilkins; 2011.

7. Blume WT, Holloway GM, Wiebe S. Temporal epileptogenesis: localizing value of scalp and subdural interictal and ictal EEG data. Epilepsia. 2001;42:508-14.

8. Pondal-Sordo M, Diosy D, Tellez-Zenteno JF, Sahjpaul R, Wiebe S. Usefulness of intracranial EEG in the decision process for epilepsy surgery. Epilepsy Res. 2007;74:176-82.

9. Blume WT, Borghesi JL, Lemieux JF. Interictal indices of temporal seizure origin. Annals of neurology 1993;34:703-9.

10. Blume WT, Young GB, Lemieux JF. EEG morphology of partial epileptic seizures. Electroencephalogr Clin Neurophysiol. 1984; 57:295-302.

11. Vera RS, Blume WT. A clinically effective spike recognition program: its use at electrocorticography. Electroencephalogr Clin Neurophysiol. 1978;45:545-8.

12. Kanazawa O, Blume WT, Girvin JP. Significance of spikes at temporal lobe electrocorticography. Epilepsia. 1996;37:50-5.

13. Lemieux JF, Blume WT. Topographical evolution of spike-wave complexes. Brain Res. 1986;373:275-87.

14. McLachlan RS, Nicholson RL, Black S, Carr T, Blume WT. Nuclear magnetic resonance imaging, a new approach to the investigation of refractory temporal lobe epilepsy. Epilepsia. 1985;26:555-62.

15. Lee DH, Gao FQ, Rogers JM, et al. MR in temporal lobe epilepsy: analysis with pathologic confirmation. Ajnr 1998;19:19-27.

16. Wong SW, Jong L, Bandur D, et al. Cortical reorganization following anterior temporal lobectomy in patients with temporal lobe epilepsy. Neurology 2009;73:518-25.

17. Mirsattari SM, Tapsell LM, Ives JR, Lee DH. MR imagingcompatible electroencephalography electrode system for an epilepsy monitoring unit. AJNR. 2008;29:1649-51.

18. Young GB. Continuous EEG monitoring in the ICU. Acta Neurol Scand. 2006;114:67-8.
19. Young GB. Nonconvulsive seizures and electroencephalogram monitoring in the intensive care unit. Adv Neurol. 2006;97: 221-7.

20. McLachlan RS, Sadler M, Pillay N, et al. Quality of life after vagus nerve stimulation for intractable epilepsy: is seizure control the only contributing factor? Eur Neurol. 2003;50:16-9.

21. Tellez-Zenteno JF, McLachlan RS, Parrent A, Kubu CS, Wiebe S. Hippocampal electrical stimulation in mesial temporal lobe epilepsy. Neurology. 2006;66:1490-4.

22. Parrent A, Almeida CS. Deep brain stimulation and cortical stimulation in the treatment of epilepsy. Adv Neurol. 2006;97: 563-72.

23. Wiebe S, Blume WT, Girvin JP, Eliasziw M. A randomized, controlled trial of surgery for temporal-lobe epilepsy. N Engl J Med. 2001;345:311-8.

24. Derry PA, Harnadek MC, McLachlan RS, Sontrop J. Influence of seizure content on interpreting psychopathology on the MMPI-2 in patients with epilepsy. J Clin Exp Neuropsychol. 1997;19: 396-404.

25. McLachlan RS, Rose KJ, Derry PA, Bonnar C, Blume WT, Girvin JP. Health-related quality of life and seizure control in temporal lobe epilepsy. Ann Neurol. 1997;41:482-9.

26. Ferro MA, Avison WR, Campbell MK, Speechley KN. Do depressive symptoms affect mothers' reports of child outcomes in children with new-onset epilepsy? Qual Life Res. 2010;19: 955-64.

27. Ferro MA, Speechley KN. Examining clinically relevant levels of depressive symptoms in mothers following a diagnosis of epilepsy in their children: a prospective analysis. Soc Psychiatry Psychiatr Epidemiol. 2011.

28. Ferro MA, Speechley KN. What about dads? An exploratory analysis of depressive symptoms in paternal primary caregivers of children with epilepsy. Epilepsy Behav. 2012;23:90-1.

29. Parrent AG, Blume WT. Stereotactic amygdalohippocampotomy for the treatment of medial temporal lobe epilepsy. Epilepsia. 1999;40:1408-16.

30. MacDougall KW, Burneo JG, McLachlan RS, Steven DA. Outcome of epilepsy surgery in patients investigated with subdural electrodes. Epilepsy Res. 2009;85:235-42.

31. Blume WT. Canadian Temporal Lobe Epilepsy Surgery Workshop: Can J Neurol Sci; 2000.

32. Blume WT, ed. Intractable Epilepsies; 2006.

33. Steven DA, Andrade-Souza YM, Burneo JG, McLachlan RS, Parrent AG. Insertion of subdural strip electrodes for the investigation of temporal lobe epilepsy. Technical note. Jo Neurosurg. 2007;106:1102-6. 